\title{
Stokes Parameters as a Minkowskian Four-vector
}

\author{
D. Han* \\ National Aeronautics and Space Administration, Goddard Space Flight Center, Code 910.1, \\ Greenbelt, Maryland 20771 \\ Y. S. $\mathrm{Kim}^{\dagger}$ \\ Department of Physics, University of Maryland, College Park, Maryland 20742 \\ Marilyn E. Noz $\ddagger$ \\ Department of Radiology, New York University, New York, New York 10016
}

\begin{abstract}
It is noted that the Jones-matrix formalism for polarization optics is a sixparameter two-by-two representation of the Lorentz group. It is shown that the four independent Stokes parameters form a Minkowskian four-vector, just like the energy-momentum four-vector in special relativity. The optical filters are represented by four-by-four Lorentz-transformation matrices. This fourby-four formalism can deal with partial coherence described by the Stokes parameters. A four-by-four matrix formulation is given for decoherence effects on the Stokes parameters, and a possible experiment is proposed. It is shown also that this Lorentz-group formalism leads to optical filters with a symmetry property corresponding to that of two-dimensional Euclidean transformations.
\end{abstract}

\section{INTRODUCTION}

There are two standard mathematical devices in polarization optics. One is the Jonesmatrix formalism [1], and the other is the set of four Stokes parameters [2, 3]. They are well-established languages in optics. In our earlier papers [4, [5], we have shown that the twoby-two Jones-matrix formalism is basically a two-by-two representation of the six-parameter Lorentz group, and the Jones vector is like the two-component spinor. This formalism allows us to use all the convenient theorems in the Lorentz group and allows us also to use optical polarizers as analog computers for the Lorentz group used in other branches of physics.

\footnotetext{
*electronic mail: han@trmm.gsfc.nasa.gov

†electronic mail: kim@umdhep.umd.edu

‡electronic mail: noz@nucmed.med.nyu.edu
} 
It is often more convenient to use the set of four numbers called the Stokes parameters. Then it is natural to write down four-by-four matrices for optical filters. The purpose of this paper is to study the parameters and the four-by-four matrices as entities in the Lorentz group. We shall show that the Stokes parameters can be grouped into a Minkowskian fourvector and the four-by-four matrices are like Lorentz transformation matrices applicable to the four-dimensional space-time.

Unlike the Jones vector, the Stokes parameters can deal with the degree of coherence between two orthogonal polarization axes. In the language of the two-by-two coherency matrix [6], the lack of coherence appears as a decrease in the magnitude of the off-diagonal elements. We shall study this decoherence effect in detail and propose an experiment to test a Lorentz effect which is mathematically equivalent to the Thomas effect in atomic spectra.

For this purpose, we start with the Stokes parameters for a purely coherent case. We then study the modification needed to deal with partially coherent polarized waves. For the purely coherent case, it is possible to construct a set of the Stokes parameters from the Jones vectors. This process is like constructing a four-vector from a pair of spinors. It is also possible to construct four-by-four filter matrices from the two-by-two Jones matrices. Thus the fourby-four matrices applicable to the four Stokes parameters form another representation of the six-parameter Lorentz group.

It is a simple matter to write down the Stokes parameters for reduced coherence. If this reduced coherence is preserved, the filter matrices are still the four-by-four matrices represented by the six-parameter Lorentz group. However, if the optical filters reduce coherence, this creates an entirely new problem. In order to deal with this case, we introduce a four-by-four "decoherence" matrix.

This new matrix is not a member of the Lorentz group, and creates a new matrix algebra. Fortunately, the four-by-four decoherence matrix can be reduced to two two-by-two matrices. One of them squeezes the first and the fourth Stokes parameters, and the other two-by-two matrix performs a two-dimensional symplectic transformation on the second and third Stokes parameters. This is quite like a Lorentz transformation in one time-like dimension and two space-like. Thus, two repeated decoherence matrices can produce a Lorentz effect similar to the Thomas effect observed in atomic spectra. This is an observable effect.

In this paper, we combine various existing mathematical devices for polarization optics into a single formalism based on the Lorentz group. This process leads to some new concepts which can be observed in laboratories. The new concepts are decoherence matrices and new optical filters.

In Sec. [1], we construct the two-by-two Jones-matrix formalism and the Stokes parameters starting from a polarized light wave. Section III summarizes our earlier papers on the Jones-matrix formalism as a representation of the Lorentz group. In Sec. IV, we show that the Stokes parameters are transformed as a Minkowskian four-vector through coherence preserving filters.

In Sec. \, we show how a Minkowskian four-vector is constructed from two spinors. It is noted that there are two different sets of spinors for the case of the Lorentz group, and this distinction is responsible for the difference between electrons and positrons. It is shown how the light wave and its complex conjugate correspond to the electron and positron respectively. In Sec. V1, we compare the Jones vectors and the Stokes parameters, and explain why the four-vector representation of the Stokes parameters contain more physics 
than the Jones-matrix formalism. It is noted in Sec. VII that the Stokes four-vector can accommodate polarized waves with partial coherence. We present in this section a matrix formulation of decoherence effects, and derive a measurable consequence.

In Sec. VIII we discuss physical implications of the procedure outlined in this paper, using a concrete physical example. In Appendix A, the Lorentz group is discussed in terms of Lorentz transformations applicable to the four-dimensional Minkowskian space. The twodimensional Euclidean group is used in our discussion of possible new optical filters. We explain this group in terms of translations and rotations on a flat plane in Appendix B.

\section{FORMULATION OF THE PROBLEM}

In studying polarized light propagating along the $z$ direction, the traditional approach is to consider the $x$ and $y$ components of the electric fields. Their amplitude ratio and the phase difference determine the degree of polarization. Thus, we can change the polarization either by adjusting the amplitudes, by changing the relative phases, or both. For convenience, we call the optical device which changes amplitudes an "attenuator" and the device which changes the relative phase a "phase shifter."

Let us write these electric fields as

$$
\left(\begin{array}{c}
E_{x} \\
E_{y}
\end{array}\right)=\left(\begin{array}{l}
A \exp \left\{i\left(k z-\omega t+\phi_{1}\right)\right\} \\
B \exp \left\{i\left(k z-\omega t+\phi_{2}\right)\right\}
\end{array}\right)
$$

where $A$ and $B$ are the amplitudes which are real and positive numbers, and $\phi_{1}$ and $\phi_{2}$ are the phases of the $x$ and $y$ components respectively. This column matrix is called the Jones vector. The content of polarization is determined by the ratio:

$$
\frac{E_{y}}{E_{x}}=\left(\frac{B}{A}\right) e^{i\left(\phi_{2}-\phi_{1}\right)}
$$

which can be written as one complex number:

$$
w=r e^{i \phi}
$$

with

$$
r=\frac{B}{A}, \quad \phi=\phi_{2}-\phi_{1}
$$

The degree of polarization is measured by these two real numbers, which are the amplitude ratio and the phase difference respectively. The transformation takes place when the light beam goes through an optical filter whose transmission properties are not isotropic.

In dealing with light waves, we have to realize that the intensity is the quantity we measure. Then there arises the question of coherence and time average. We are thus led to consider the following parameters.

$$
\begin{array}{ll}
S_{11}=<E_{x}^{*} E_{x}>, & S_{22}=<E_{y}^{*} E_{y}>, \\
S_{12}=<E_{x}^{*} E_{y}>, & S_{21}=<E_{y}^{*} E_{x}>.
\end{array}
$$

Then, we are naturally invited to write down the two-by-two matrix: 


$$
C=\left(\begin{array}{cc}
<E_{x}^{*} E_{x}> & <E_{y}^{*} E_{x}> \\
<E_{x}^{*} E_{y}> & <E_{y}^{*} E_{y}>
\end{array}\right),
$$

where $\left\langle E_{i}^{*} E_{j}>\right.$ is the time average of $E_{i}^{*} E_{j}$. The above form is called the coherency matrix [6].

It is sometimes more convenient to use the following combinations of parameters.

$$
\begin{aligned}
& S_{0}=S_{11}+S_{22}, \\
& S_{1}=S_{11}-S_{22} \\
& S_{2}=S_{12}+S_{21} \\
& S_{3}=-i\left(S_{12}-S_{21}\right),
\end{aligned}
$$

These four parameters are called the Stokes parameters in the literature [2,3].

The purpose of the present paper is to show that these four parameters form a Minkowskian four-vector when the light wave goes through optical filters. Once the four Stokes parameters are introduced, it is quite natural to construct a four-dimensional vector and transformation matrices applicable to this vector. These four-by-four matrices are called Mueller matrices [2]. In this paper, we shall therefore show that the Mueller matrices are like four-by-four Lorentz transformation matrices.

G. G. Stokes was an active researcher during the 19th Century [2]. The mathematics of the Stokes parameters is a very old science, and there are many interesting mathematical devices. For instance, we can define a three-dimensional Cartesian coordinate system spanned by $S_{1}, S_{2}, S_{3}$. We can then consider a sphere whose maximum radius is $S_{0}$. This is called the Poincaré sphere and is the standard geometrical language for the Stokes parameters [6]. We shall use the geometry of this sphere in Secs. VI and VII of this paper.

\section{JONES-MATRIX FORMALISM}

We presented in our earlier papers [4], the Jones-matrix formalism as a representation of the Lorentz group. We used there the concept of squeeze transformations to describe attenuation filters. Let us summarize in this section the Lorentz-group content of this formalism which will be useful for discussing the Stokes parameters.

There are two transverse directions which are perpendicular to each other. The absorption coefficient in one transverse direction could be different from the coefficient along the other direction. Thus, there is the "polarization" coordinate in which the absorption can be described by

$$
\left(\begin{array}{cc}
e^{-\eta_{1}} & 0 \\
0 & e^{-\eta_{2}}
\end{array}\right)=e^{-\left(\eta_{1}+\eta_{2}\right) / 2}\left(\begin{array}{cc}
e^{\eta / 2} & 0 \\
0 & e^{-\eta / 2}
\end{array}\right)
$$

with $\eta=\eta_{2}-\eta_{1}$. This attenuation matrix tells us that the electric fields are attenuated at two different rates. The exponential factor $e^{-\left(\eta_{1}+\eta_{2}\right) / 2}$ reduces both components at the same rate and does not affect the degree of polarization. The effect of polarization is solely determined by the attenuation matrix

$$
A(0, \eta)=\left(\begin{array}{cc}
e^{\eta / 2} & 0 \\
0 & e^{-\eta / 2}
\end{array}\right)
$$


This type of mathematical operation is quite familiar to us from squeezed states of light and from Lorentz boosts of spinors. We call the above matrix the "attenuator."

Another basic element is the optical filter with different values of the index of refraction along the two orthogonal directions. The effect of this filter can be written as

$$
\left(\begin{array}{cc}
e^{i \delta_{1}} & 0 \\
0 & e^{i \delta_{2}}
\end{array}\right)=e^{-i\left(\delta_{1}+\delta_{2}\right) / 2}\left(\begin{array}{cc}
e^{-i \delta / 2} & 0 \\
0 & e^{i \delta / 2}
\end{array}\right)
$$

with $\delta=\delta_{2}-\delta_{1}$. In measurement processes, the overall phase factor $e^{-i\left(\delta_{1}+\delta_{2}\right) / 2}$ cannot be detected, and can therefore be deleted. The polarization effect of the filter is solely determined by the matrix

$$
P(0, \delta)=\left(\begin{array}{cc}
e^{-i \delta / 2} & 0 \\
0 & e^{i \delta / 2}
\end{array}\right)
$$

This form was noted as one of the basic components of the Jones-matrix formalism in Sec. [I]. This phase-shifter matrix appears like a rotation matrix around the $z$ axis in the theory of rotation groups, but it plays a different role in this paper. We shall hereafter call this matrix a phase shifter.

The polarization axes are not always the $x$ and $y$ axes. For this reason, we need the rotation matrix

$$
R(\theta)=\left(\begin{array}{cc}
\cos (\theta / 2) & -\sin (\theta / 2) \\
\sin (\theta / 2) & \cos (\theta / 2)
\end{array}\right)
$$

If the polarization coordinate is rotated by an angle $\theta / 2$, the attenuator and phase-shifter of Eq.(3.2) and Eq.(3.4) respectively become

$$
\begin{aligned}
A(\theta, \eta) & =R(\theta) A(0, \eta) R(-\theta) \\
& =\left(\begin{array}{cc}
e^{\eta / 2} \cos ^{2}(\theta / 2)+e^{-\eta / 2} \sin ^{2}(\theta / 2) & \left(e^{\eta / 2}-e^{-\eta / 2}\right) \cos (\theta / 2) \sin (\theta / 2) \\
\left(e^{\eta / 2}-e^{-\eta / 2}\right) \cos (\theta / 2) \sin (\theta / 2) & e^{-\eta / 2} \cos ^{2}(\theta / 2)+e^{\eta / 2} \sin ^{2}(\theta / 2)
\end{array}\right),
\end{aligned}
$$

and

$$
\begin{aligned}
P(\theta, \delta) & =R(\theta) P(0, \delta) R(-\theta) \\
& =\left(\begin{array}{ll}
e^{-i \delta / 2} \cos ^{2}(\theta / 2)+e^{i \delta / 2} \sin ^{2}(\theta / 2) & \left(e^{-i \delta / 2}-e^{i \delta / 2}\right) \cos (\theta / 2) \sin (\theta / 2) \\
\left(e^{-i \delta / 2}-e^{i \delta / 2}\right) \cos (\theta / 2) \sin (\theta / 2) & e^{i \delta / 2} \cos ^{2}(\theta / 2)+e^{-i \delta / 2} \sin ^{2}(\theta / 2)
\end{array}\right) .
\end{aligned}
$$

The Jones-matrix formalism consists of repeated applications of the three basic operations. In order to study this more systematically, let us use the generators of these transformations. First, the rotation matrix of Eq.(3.5) takes the form:

$$
R(\theta)=\exp \left(-i \theta J_{2}\right),
$$

with

$$
J_{2}=\frac{1}{2}\left(\begin{array}{cc}
0 & -i \\
i & 0
\end{array}\right)
$$


The attenuation operator of Eq.(3.2) can also be written in the exponential form:

$$
A(0, \eta)=\exp \left(-i \eta K_{3}\right)
$$

with

$$
K_{3}=\frac{i}{2}\left(\begin{array}{cc}
1 & 0 \\
0 & -1
\end{array}\right) .
$$

If we take the commutation relation between the above generators, we end up with another generator:

$$
\left[J_{2}, K_{3}\right]=i K_{1}
$$

with

$$
K_{1}=\frac{i}{2}\left(\begin{array}{ll}
0 & 1 \\
1 & 0
\end{array}\right)
$$

We are then led to write the commutation relations:

$$
\left[K_{3}, K_{1}\right]=-i J_{2}, \quad\left[K_{1}, J_{2}\right]=i K_{3}
$$

These three generators indeed form a closed set of commutation relations. The threeparameter groups generated by this set of commutation relations are $O(2,1), S p(2, r)$, and $S U(1,1)$. The group $S U(1,1)$ has been extensively discussed in the literature in connection with squeezed states of light [7]. The group $O(2,1)$ is the Lorentz group applicable to one time-like dimension and two space-like dimensions. The group $S p(2, r)$ is the basic symmetry group for the Wigner function for one-mode squeezed states [7],8]. The relevance of this group in polarization optics has also been noted in the literature [4, 9, 10].

The phase-shifter of Eq.(3.4) can be written as

$$
P(0, \delta)=\exp \left(-i \delta J_{3}\right)
$$

with

$$
J_{3}=\frac{1}{2}\left(\begin{array}{cc}
1 & 0 \\
0 & -1
\end{array}\right)
$$

If we take a commutation relation of this operator with $J_{2}$,

$$
\left[J_{3}, J_{2}\right]=i J_{1},
$$

with

$$
J_{1}=\frac{1}{2}\left(\begin{array}{ll}
0 & 1 \\
1 & 0
\end{array}\right)
$$

These three generators satisfy the commutation relations

$$
\left[J_{i}, J_{j}\right]=i \epsilon_{i j k} J_{k}
$$

This set of commutations relations is very familiar to us. They generate the threedimensional rotation group and the $S U(2)$ group governing rotational symmetry of spin-1/2 particles. These are also three-parameter groups. 
If we apply both attenuators and phase-shifters in random order in laboratories, the corresponding mathematics is to combine the two three-parameter groups by mixing up their commutation relations. The resulting closed set of commutation relations consists of [四]

$$
\left[J_{i}, K_{j}\right]=i \epsilon_{i j k} K_{k}, \quad\left[K_{i}, J_{j}\right]=-i \epsilon_{i j k} J_{k}
$$

in addition to the set given in Eq.(3.16).

These are the six generators for the two-by-two representation of the Lorentz group which is often called $S L(2, c)$. This group is the standard language in elementary particle physics dealing with spin-1/2 particles. In optics, the Lorentz group gained its prominence recently in connection with squeezed states of light [7]. It is quite natural for us to study the two-by-two Jones matrices and four-by-four Mueller matrices within the framework of the Lorentz group.

\section{TRANSFORMATION PROPERTIES OF THE STOKES PARAMETERS}

In order to study the effect of each filter, let us note that the effect of the attenuator of Eq.(3.2) on the incoming light of Eq.(2.1) is

$$
\left(\begin{array}{c}
e^{\eta / 2} E_{x} \\
e^{-\eta / 2} E_{y}
\end{array}\right)
$$

The effect of the phase shifter of Eq.(3.4) on the incoming light of Eq.(2.1) is

$$
\left(\begin{array}{c}
e^{-i \delta / 2} E_{x} \\
e^{i \delta / 2} E_{y}
\end{array}\right)
$$

The effect of the rotation matrix of Eq.(3.5) on the incoming light wave is

$$
\left(\begin{array}{c}
(\cos (\theta / 2)) E_{x}-(\sin (\theta / 2)) E_{y} \\
(\sin (\theta / 2)) E_{x}+(\cos (\theta / 2)) E_{y}
\end{array}\right)
$$

Under the rotation of Eq.(3.5) which transforms the incoming wave of Eq.(2.1) to Eq.(4.3), $S_{11}$ and $S_{22}$ become

$$
\frac{\cos \theta}{2}\left(S_{11}-S_{22}\right)-\frac{\sin \theta}{2}\left(S_{12}+S_{21}\right)+\frac{1}{2}\left(S_{11}+S_{22}\right)
$$

and

$$
-\frac{\cos \theta}{2}\left(S_{11}-S_{22}\right)+\frac{\sin \theta}{2}\left(S_{12}+S_{21}\right)+\frac{1}{2}\left(S_{11}+S_{22}\right)
$$

respectively. It is clear from these expressions that $\left(S_{11}+S_{22}\right)$ is invariant under this rotation. As for $\left(S_{11}-S_{22}\right)$, the rotation leads to

$$
\cos \theta\left(S_{11}-S_{22}\right)-\sin \theta\left(S_{12}+S_{21}\right) .
$$

Under the same rotation, $S_{12}$ and $S_{21}$ become 


$$
\frac{\sin \theta}{2}\left(S_{11}-S_{22}\right)+\frac{\cos \theta}{2}\left(S_{12}+S_{21}\right)+\frac{1}{2}\left(S_{12}-S_{21}\right),
$$

and

$$
\frac{\sin \theta}{2}\left(S_{11}-S_{22}\right)+\frac{\cos \theta}{2}\left(S_{12}+S_{21}\right)-\frac{1}{2}\left(S_{12}-S_{21}\right),
$$

respectively. Thus $\left(S_{12}-S_{21}\right)$ remains invariant, while $\left(S_{12}+S_{21}\right)$ becomes

$$
\sin \theta\left(S_{11}-S_{22}\right)+\cos \theta\left(S_{12}+S_{21}\right) .
$$

We can thus write the effect of the rotation as

$$
\left(\begin{array}{cc}
\cos \theta & -\sin \theta \\
\sin \theta & \cos \theta
\end{array}\right)\left(\begin{array}{c}
S_{11}-S_{22} \\
S_{12}+S_{21}
\end{array}\right) .
$$

Under the phase-shift transformation of Eq.(3.4) which leads to Eq.(4.2), $S_{11}$ and $S_{22}$ remain invariant, while $S_{12}$ and $S_{21}$ become $e^{i \delta} S_{12}$ and $e^{-i \delta} S_{21}$. The result is

$$
\left(\begin{array}{cc}
\cos \delta & -\sin \delta \\
\sin \delta & \cos \delta
\end{array}\right)\left(\begin{array}{c}
S_{12}+S_{21} \\
-i\left(S_{12}-S_{21}\right)
\end{array}\right) .
$$

Under the squeeze transformation of Eq.(3.2) which leads to Eq.(4.1), $S_{11}$ and $S_{22}$ become $e^{\eta} S_{11}$ and $e^{-\eta} S_{22}$ respectively, while $S_{12}$ and $S_{21}$ remain unchanged. This can be translated into

$$
\left(\begin{array}{cc}
\cosh \eta & \sinh \eta \\
\sinh \eta & \cosh \eta
\end{array}\right)\left(\begin{array}{c}
S_{11}+S_{22} \\
S_{11}-S_{22}
\end{array}\right) .
$$

Thus in terms of the Stokes parameters given in Eq.(2.6), we can write the above three transformations as

$$
\begin{aligned}
& \left(\begin{array}{cccc}
1 & 0 & 0 & 0 \\
0 & \cos \theta & -\sin \theta & 0 \\
0 & \sin \theta & \cos \theta & 0 \\
0 & 0 & 0 & 1
\end{array}\right)\left(\begin{array}{l}
S_{0} \\
S_{1} \\
S_{2} \\
S_{3}
\end{array}\right), \\
& \left(\begin{array}{cccc}
1 & 0 & 0 & 0 \\
0 & 1 & 0 & 0 \\
0 & 0 & \cos \delta & -\sin \delta \\
0 & 0 & \sin \delta & \cos \delta
\end{array}\right)\left(\begin{array}{l}
S_{0} \\
S_{1} \\
S_{2} \\
S_{3}
\end{array}\right),
\end{aligned}
$$

and

$$
\left(\begin{array}{cccc}
\cosh \eta & \sinh \eta & 0 & 0 \\
\sinh \eta & \cosh \eta & 0 & 0 \\
0 & 0 & 1 & 0 \\
0 & 0 & 0 & 1
\end{array}\right)\left(\begin{array}{c}
S_{0} \\
S_{1} \\
S_{2} \\
S_{3}
\end{array}\right)
$$

The above three matrices are generated by $J_{3}, J_{1}$ and $K_{1}$ respectively, where 


$$
J_{3}=\left(\begin{array}{cccc}
0 & 0 & 0 & 0 \\
0 & 0 & -i & 0 \\
0 & i & 0 & 0 \\
0 & 0 & 0 & 0
\end{array}\right), \quad J_{1}=\left(\begin{array}{cccc}
0 & 0 & 0 & 0 \\
0 & 0 & 0 & 0 \\
0 & 0 & 0 & -i \\
0 & 0 & i & 0
\end{array}\right)
$$

and

$$
K_{1}=\left(\begin{array}{cccc}
0 & i & 0 & 0 \\
i & 0 & 0 & 0 \\
0 & 0 & 0 & 0 \\
0 & 0 & 0 & 0
\end{array}\right)
$$

The commutation relation between $J_{3}$ and $J_{1}$ leads to another generator $J_{2}$, with

$$
J_{2}=\left(\begin{array}{cccc}
0 & 0 & 0 & 0 \\
0 & 0 & 0 & i \\
0 & 0 & 0 & 0 \\
0 & -i & 0 & 0
\end{array}\right)
$$

These three matrices generate the three-dimensional rotation group with the closed set of commutation relations.

$$
\left[J_{i}, J_{j}\right]=i \epsilon_{i j k} J_{k}
$$

If we take commutations relations of $K_{1}$ with these rotation generators, we end up with two additional generators $K_{2}$ and $K_{3}$, where

$$
K_{2}=\left(\begin{array}{cccc}
0 & 0 & i & 0 \\
0 & 0 & 0 & 0 \\
i & 0 & 0 & 0 \\
0 & 0 & 0 & 0
\end{array}\right), \quad K_{3}=\left(\begin{array}{cccc}
0 & 0 & 0 & i \\
0 & 0 & 0 & 0 \\
0 & 0 & 0 & 0 \\
i & 0 & 0 & 0
\end{array}\right)
$$

These three $K_{i}$ generators satisfy the commutation relations

$$
\left[K_{i}, K_{j}\right]=-i \epsilon_{i j k} J_{k}
$$

The right-hand side of the above expression is not $K_{i}$ but $J_{i}$. Thus, in order to get a closed set of commutators, we have to take commutation relations between $J_{i}$ and $K_{i}$. The result is

$$
\left[J_{i}, K_{j}\right]=i \epsilon_{i j k} K_{k}
$$

Thus the six matrices consisting of three $J$ and three $K$ matrices form a closed set of a group of transformations applicable to four-dimensional space.

If the above transformation group is applied to the four-dimensional space-time coordinate $(t, z, x, y)$, it becomes the group of Lorentz transformations in the Minkowskian space. In deriving the above three sets of commutation relations, we have not used any of the principles of special relativity. The commutation relations are derived strictly from the properties of the optical filters. The Stokes parameters have nothing to do with special relativity. Yet, it is remarkable that they can be formulated in terms of the mathematics of Lorentz transformations. 


\section{SPINORS AND FOUR-VECTORS IN THE LORENTZ GROUP}

We are now confronted with the question of why the Stokes parameters have to behave like a Minkowskian four-vector. For this purpose, let us go back to Sec. IIII and consider repeated applications of the three basic operations. We shall see first whether the two-bytwo matrix algebra of Sec. IIII can be represented as a representation of the six-parameter Lorentz group. We shall then investigate whether the four-by-four matrices of Sec. IV can be systematically obtained from the Jones matrices.

For this purpose, let us start with the three Pauli spin matrices of the form

$$
\sigma_{1}=\left(\begin{array}{cc}
0 & 1 \\
1 & 0
\end{array}\right), \quad \sigma_{2}=\left(\begin{array}{cc}
0 & -i \\
i & 0
\end{array}\right), \quad \sigma_{3}=\left(\begin{array}{cc}
1 & 0 \\
0 & -1
\end{array}\right) .
$$

Then, the rotation generators

$$
J_{i}=\frac{1}{2} \sigma_{i}
$$

satisfy the closed set of commutation relations of Eq.(4.19).

We can also construct three boost generators

$$
K_{i}=\frac{i}{2} \sigma_{i}
$$

which satisfy the commutation relations given in Eq.(4.21). The $K_{i}$ matrices alone do not form a closed set of commutation relations, their commutation relations with the rotation generators are given in Eq. 4.22).

The six matrices $J_{i}$ and $K_{i}$ form a closed set of commutation relations, and they are like the generators of the Lorentz group applicable to the $(3+1)$-dimensional Minkowski space. The group generated by the above six matrices is called $S L(2, c)$ consisting of all two-by-two complex matrices with unit determinant.

In order to construct four-vectors, we need two different spinor representations of the Lorentz group. Let us go to the commutation relations for the generators given in Eqs.(4.19), (4.21) and (4.22). These commutators are not invariant under the sign change of the rotation generators $J_{i}$, but are invariant under the sign change of the squeeze generators $K_{i}$. Thus, to each spinor representation, there is another representation with the squeeze generators with opposite sign. This allows us to construct another representation with the generators:

$$
\dot{J}_{i}=\frac{1}{2} \sigma_{i}, \quad \dot{K}_{i}=\frac{-i}{2} \sigma_{i} .
$$

We call this representation the "dotted" representation.

There are therefore two different sets of Lorentz transformation matrices. If we write the most general form of the transformation matrix using undotted generators, it takes the form

$$
L=\exp \left\{-\frac{i}{2} \sum_{i=1}^{3}\left(\theta_{i} \sigma_{i}+i \eta_{i} \sigma_{i}\right)\right\} .
$$

Then the transformation matrix in the dotted representation becomes 


$$
\dot{L}=\exp \left\{-\frac{i}{2} \sum_{i=1}^{3}\left(\theta_{i} \sigma_{i}-i \eta_{i} \sigma_{i}\right)\right\}
$$

In both of the above matrices, the Hermitian conjugation changes the direction of rotation. However, it does not change the direction of boosts. We can achieve this only by interchanging $L$ to $\dot{L}$, and we shall call this the "dot" conjugation.

Likewise, there are two different sets of spinors. Let us use $u$ and $v$ for the up and down spinors for "undotted" representation. Then $\dot{u}$ and $\dot{v}$ are for the dotted representation. The four-vectors are then constructed as [1]

$$
\begin{aligned}
& u \dot{u}=-(x-i y), \quad v \dot{v}=(x+i y), \\
& u \dot{v}=(t+z), \quad v \dot{u}=-(t-z) .
\end{aligned}
$$

The relation between the $S L(2, c)$ spinors and the four-vectors has been discussed in the literature [11 13]. It is possible to construct the four-vector with the four $S L(2, c)$ spinors 12, 14. Indeed,

$$
\begin{aligned}
-u \dot{u} & =(1, i, 0,0), & v \dot{v}=(1,-i, 0,0), \\
u \dot{v} & =(0,0,1,1), & v \dot{v}_{+}=(0,0,1,-1) .
\end{aligned}
$$

It is possible to construct a six-component Maxwell tensor by making combinations of two undotted and dotted spinors [12]. For massless particles, the only gauge-invariant components are $u u$ and $\dot{v} \dot{v}$ [15]. They correspond to the photons in the Maxwell tensor representation with positive and negative helicities respectively. It is also possible to construct Maxwell-tensor fields only for a massive particle, and obtain massless Maxwell fields by group contraction [16].

$$
C=\left(\begin{array}{ll}
u \dot{v} & -u \dot{u} \\
v \dot{v} & -v \dot{u}
\end{array}\right)=\left(\begin{array}{l}
u \\
v
\end{array}\right)\left(\begin{array}{ll}
\dot{v} & -\dot{u}
\end{array}\right)
$$

where $u$ and $\dot{u}$ are one if the spin is up, and are zero if the spin is down, while $v$ and $\dot{v}$ are zero and one for the spin-up and spin-down cases. The transformation matrix applicable to the column vector in the above expression is the two-by-two matrix given in Eq.(5.5). What is then the transformation matrix applicable to the row vector $(\dot{v},-\dot{u})$ from the right-hand side? It is the transpose of the matrix applicable to the column vector $(\dot{v},-\dot{u})$. We can obtain this column vector from

$$
\left(\begin{array}{c}
\dot{v} \\
-\dot{u}
\end{array}\right)
$$

by applying to it the matrix

$$
g=-i \sigma_{2}=\left(\begin{array}{cc}
0 & -1 \\
1 & 0
\end{array}\right)
$$

This matrix also has the property

$$
g \sigma_{i} g^{-1}=-\left(\sigma_{i}\right)^{T},
$$


where the superscript $T$ means the transpose of the matrix. The transformation matrix applicable to the column vector of Eq.(5.10) is $\dot{L}$ of Eq.(5.6). Thus the matrix applicable to the row vector $(\dot{v},-\dot{u})$ in Eq.(5.9) is

$$
\left\{g^{-1} \dot{L} g\right\}^{T}=g^{-1} \dot{L}^{T} g .
$$

This is precisely the Hermitian conjugate of $L$.

Let us now consider its transformation properties. The matrix of Eq.(2.5) is like

$$
C=\left(\begin{array}{cc}
t+z & x-i y \\
x+i y & t-z
\end{array}\right),
$$

where the set of variables $(x, y, z, t)$ is transformed like a four-vector under Lorentz transformations. Furthermore, it is known that the Lorentz transformation of this four-vector is achieved through the formula

$$
C^{\prime}=L C L^{\dagger},
$$

where the transformation matrix $L$ is that of Eq.(5.5). The construction of four-vectors from the two-component spinors is not a trivial task [11,16]. The two-by-two representation of Eq.(5.14) requires one more step of complication.

We are not the first ones to suspect that the coherency matrix behaves like a four-vector. This was done by Barakat in 1963 [17]. However, Sec. D shows that we need two different two-by-two representations of the Lorentz group to establish the connection between the Jones vectors and the Stokes parameters in a covariant manner. The two different spinors are the column vectors of $(u, v)$ and $(\dot{u}, \dot{v})$.

This paper allows us to associate these spinors as

$$
\left(\begin{array}{l}
u \\
v
\end{array}\right)=\left(\begin{array}{l}
E_{x} \\
E_{y}
\end{array}\right),
$$

and

$$
\left(\begin{array}{c}
\dot{u} \\
\dot{v}
\end{array}\right)=\left(\begin{array}{c}
-E_{y}^{*} \\
E_{x}^{*}
\end{array}\right) .
$$

The symmetry between the dotted and undotted representations is responsible for the electron-positron symmetry in the Dirac equation [12]. It is interesting to note that this symmetry is applicable also to the polarization vectors of Eq.(5.16) and Eq.(5.17).

\section{JONES SPINORS AND STOKES VECTORS}

The Jones vector is a two-component vector in the conventional formalism. Since, however, it is like a spinor in the Lorentz group, we call it hereafter the Jones spinor. The Jones spinor and the Stokes four-vector are two different representations of the same Lorentz group. Why do we construct two different representations? The difference is in physics.

Since the four-vector contains more elements than the two-component spinor, the Stokes vector should give more information than the Jones spinor. This is translated into the 
invariance properties of Stokes parameters. As the four-scalar $\left(t^{2}-z^{2}-x^{2}-y^{z}\right)$ is invariant under Lorentz transformations, the quantity

$$
S^{2}=S_{0}^{2}-S_{1}^{2}-S_{2}^{2}-S_{3}^{2}
$$

remains invariant under filtering processes discussed in this paper. We shall hereafter call this quantity "Stokes scalar." If the Stokes Scalar is zero, the system is completely coherent. This scalar quantity is positive if the system is partially coherent.

Indeed, this degree of coherence is what the Stokes vector can tell while the Jones spinor cannot. If the filter system leaves the Stokes scalar invariant, it is a coherence-preserving system. This quantity is not preserved if the filters cause random variations of phases. The best way to describe this degree of coherence is to construct a Poincare sphere in the three-dimensional space of $S_{1}, S_{2}$ and $S_{3}$. The radius of this sphere is

$$
R=\left(S_{1}^{2}+S_{2}^{2}+S_{3}^{2}\right)^{1 / 2}
$$

Then the ratio $R / S_{0}$ gives the degree of coherence.

This radius takes the maximum value $S_{0}$ when the system is completely coherent, and it takes the minimum value of $S_{1}$ when the system is completely incoherent. In this minimum case, both $S_{2}$ and $S_{3}$ vanish. The question then is whether there is a four-by-four matrix which reduces these two components. If so, how can this matrix be augmented to the set of transformation matrices discussed in Sec. IV?

\section{DECOHERENCE MATRICES}

Let us go back to the four-by-four representation of Sec. IV. For the Stokes four-vector, we can translate the two-by-two attenuator Eq.(3.2) applicable to the Jones vector into the four-by-four matrix

$$
A(0, \eta)=\left(\begin{array}{cccc}
\cosh \eta & \sinh \eta & 0 & 0 \\
\sinh \eta & \cosh \eta & 0 & 0 \\
0 & 0 & 1 & 0 \\
0 & 0 & 0 & 1
\end{array}\right)
$$

Likewise, the phase-shifter of Eq.(3.4) is translated into

$$
P(0, \delta)=\left(\begin{array}{cccc}
1 & 0 & 0 & 0 \\
0 & 1 & 0 & 0 \\
0 & 0 & \cos \delta & -\sin \delta \\
0 & 0 & \sin \delta & \cos \delta
\end{array}\right)
$$

The rotation matrix of Eq.(3.5) becomes

$$
R(\theta)=\left(\begin{array}{cccc}
1 & 0 & 0 & 0 \\
0 & \cos \theta & -\sin \theta & 0 \\
0 & \sin \theta & \cos \theta & 0 \\
0 & 0 & 0 & 1
\end{array}\right)
$$


If the two transverse components lose coherence, the time-averaged values $S_{12}$ and $S_{21}$ become smaller. We can therefore use the matrix

$$
\left(\begin{array}{cccc}
1 & 0 & 0 & 0 \\
0 & 1 & 0 & 0 \\
0 & 0 & e^{-2 \lambda} & 0 \\
0 & 0 & 0 & e^{-2 \lambda}
\end{array}\right)
$$

which can also be written as

$$
e^{-\lambda}\left(\begin{array}{cccc}
e^{\lambda} & 0 & 0 & 0 \\
0 & e^{\lambda} & 0 & 0 \\
0 & 0 & e^{-\lambda} & 0 \\
0 & 0 & 0 & e^{-\lambda}
\end{array}\right)
$$

where $e^{-\lambda}$ is the overall decoherence factor. For convenience, we define the decoherence matrix as

$$
D(\lambda)=\left(\begin{array}{cccc}
e^{\lambda} & 0 & 0 & 0 \\
0 & e^{\lambda} & 0 & 0 \\
0 & 0 & e^{-\lambda} & 0 \\
0 & 0 & 0 & e^{-\lambda}
\end{array}\right)
$$

which is generated by

$$
Q_{3}=\left(\begin{array}{cccc}
i & 0 & 0 & 0 \\
0 & i & 0 & 0 \\
0 & 0 & -i & 0 \\
0 & 0 & 0 & -i
\end{array}\right)
$$

The introduction of the above matrix into the existing set of six generators of the Lorentz group leads to the fifteen parameter group of $O(3,3)$ or $S L(4, r)$ [18], and this is beyond the scope of the present paper. This however does not prevent us from looking for an interesting subgroup which will play the key role in accommodating the decoherence matrix into the four-by-four matrix formalism for the Stokes parameters.

It is interesting to see that this decoherence matrix commutes with the attenuator of Eq.(7.1) and the phase-shifter of Eq.(7.2), but it does not commute with the rotator of Eq.(7.3). Thus, the complication is reduced to the non-commuting algebra of this rotation matrix and the decoherence matrix. As is given in Eq.4.16), the generator of $R(\theta)$ of Eq.(7.3) takes the form

$$
J_{3}=\left(\begin{array}{cccc}
0 & 0 & 0 & 0 \\
0 & 0 & -i & 0 \\
0 & i & 0 & 0 \\
0 & 0 & 0 & 0
\end{array}\right)
$$

If we take the commutator of this matrix with the generator of $Q_{3}$,

$$
\left[J_{3}, Q_{3}\right]=2 i W_{3}
$$


with

$$
W_{3}=\left(\begin{array}{cccc}
0 & 0 & 0 & 0 \\
0 & 0 & i & 0 \\
0 & i & 0 & 0 \\
0 & 0 & 0 & 0
\end{array}\right)
$$

In order to see the physics of these matrices, let us go to the Poincaré sphere of this system. In the three-dimensional space with the three Cartesian coordinate variable $S_{1}, S_{2}$ and $S_{3}$, rotations around the $S_{3}$ axis generated by $J_{3}$ do not change the first and the last components of the Stokes four-vector $\left(S_{0}, S_{1}, S_{2}, S_{3}\right)$. We can thus divide this fourcomponent vector into two two-component vectors:

$$
S_{A}=\left(\begin{array}{c}
S_{0} \\
S_{3}
\end{array}\right), \quad S_{B}=\left(\begin{array}{c}
S_{1} \\
S_{2}
\end{array}\right)
$$

The effect of the decoherence matrix on $S_{A}$ will be

$$
\left(\begin{array}{cc}
e^{\lambda} & 0 \\
0 & e^{-\lambda}
\end{array}\right)\left(\begin{array}{l}
S_{0} \\
S_{3}
\end{array}\right)
$$

This is a squeeze transformation not affected by rotations around the $S_{3}$ axis. If we take into account the overall factor mentioned after Eq.(7.5), the effect of decoherence on $S_{A}$ is

$$
\left(\begin{array}{cc}
1 & 0 \\
0 & e^{-2 \lambda}
\end{array}\right)\left(\begin{array}{l}
S_{0} \\
S_{3}
\end{array}\right)
$$

and this expression is invariant under rotations generated by $J_{3}$. Under repeated applications, the matrix algebra is simply

$$
\left(\begin{array}{cc}
1 & 0 \\
0 & e^{-2 \rho}
\end{array}\right)\left(\begin{array}{cc}
1 & 0 \\
0 & e^{-2 \lambda}
\end{array}\right)\left(\begin{array}{l}
S_{0} \\
S_{3}
\end{array}\right)
$$

The story is quite different for $S_{B}$. Here we are dealing with rotations and squeeze transformations in the two-dimensional space of $S_{1}$ and $S_{2}$. In order to take advantage of the mathematics of squeezed states [0], let us write $Q_{3}, W_{3}, J_{3}$ as

$$
B_{1}=\frac{1}{2} Q_{3}, \quad B_{2}=\frac{1}{2} W_{3}, \quad L_{3}=\frac{1}{2} J_{3} .
$$

These matrices form the closed set of commutation relations

$$
\left[B_{1}, B_{2}\right]=-i L_{3}, \quad\left[B_{2}, L_{3}\right]=i B_{3}, \quad\left[L_{3}, B_{1}\right]=i B_{2},
$$

which are very familiar to us from the squeezed state of light. They generate the group $S p(2)$ or $S U(1,1)$. This is by now a standard mathematical tool in optics. The algebraic property of this group is the same as the group of Lorentz transformations in two spacelike dimensions and one time-like dimension. This group is routinely called $O(2,1)$ in the literature, and the generators satisfy the same set of commutation relations as the above set for $\operatorname{Sp}(2)[7]$. 
Thus the matrix algebra applicable to the two-component vector $S_{B}$ is the same as that for the $S p(2)$ squeezed states and/or the $(2+1)$-dimensional Lorentz group. The decoherence along the $S_{1}$ direction is

$$
\left(\begin{array}{cc}
e^{\lambda} & 0 \\
0 & e^{-\lambda}
\end{array}\right)
$$

The decoherence transformation along the direction which makes an angle $\theta$ with the $S_{1}$ axis is

$$
\left(\begin{array}{cc}
\cosh \rho+(\sinh \rho) \cos (2 \theta) & (\sinh \rho) \sin (2 \theta) \\
(\sinh \rho) \sin (2 \theta) & \cosh \rho-(\sinh \rho) \cos (2 \theta)
\end{array}\right) .
$$

Thus the decoherence along the $S_{1}$ direction followed by the above transformation is

$$
\left(\begin{array}{cc}
\cosh \rho+(\sinh \rho) \cos (2 \theta) & (\sinh \rho) \sin (2 \theta) \\
(\sinh \rho) \sin (2 \theta) & \cosh \rho-(\sinh \rho) \cos (2 \theta)
\end{array}\right)\left(\begin{array}{cc}
e^{\lambda} & 0 \\
0 & e^{-\lambda}
\end{array}\right) .
$$

The computation of this matrix algebra leads to another decoherence matrix preceded by a rotation matrix

$$
\left(\begin{array}{cc}
\cosh \xi+(\sinh \xi) \cos (2 \alpha) & (\sinh \xi) \sin (2 \alpha) \\
(\sinh \xi) \sin (2 \alpha) & \cosh \xi-(\sinh \xi) \cos (2 \alpha)
\end{array}\right)\left(\begin{array}{cc}
\cos \phi & -\sin \phi \\
\sin \phi & \cos \phi
\end{array}\right)
$$

where

$$
\begin{aligned}
\cosh \xi & =(\cosh \lambda) \cosh \rho+(\sinh \lambda)(\sinh \rho) \cos \theta \\
\tan \alpha & =\frac{(\sin \theta)[\sinh \rho+(\tanh \lambda)(\cosh \rho-1) \cos \theta]}{(\sinh \rho) \cos \theta+(\tanh \lambda)\left[1+(\cosh \rho-1)(\cos \theta)^{2}\right.} \\
\tan \phi & =\frac{(\tanh \rho)(\tan \lambda) \sin 2 \theta}{1+(\tanh \lambda)(\tan \rho) \cos 2 \theta}
\end{aligned}
$$

The calculation leading to the above expression is well known from the squeezed state and the Lorentz transformation. The overall decoherence factors for Eq.(7.17) and Eq.(7.18) are $e^{-\lambda}$ and $e^{-\rho}$ respectively. The overall factor for Eq. (7.20) is $e^{-\xi}$, and the net decoherence effect on $S_{B}$ is

$$
e^{-\xi}\left(\begin{array}{cc}
\cosh \xi+(\sinh \xi) \cos (2 \alpha) & (\sinh \xi) \sin (2 \alpha) \\
(\sinh \xi) \sin (2 \alpha) & \cosh \xi-(\sinh \xi) \cos (2 \alpha)
\end{array}\right)\left(\begin{array}{cc}
\cos \phi & -\sin \phi \\
\sin \phi & \cos \phi
\end{array}\right) .
$$

The non-trivial aspect of this calculation is the rotation matrix in the above expression. The decoherence followed by another decoherence does not always result in a decoherence. It is a decoherence preceded by a rotation. It is a simple matter to detect this rotation once the decoherence filters are built in laboratories.

This effect of the Lorentz group has been discussed in connection with polarization optics [9,10]. In special relativity, this extra rotation is called the Thomas effect and manifests itself in the energy spectrum of the hydrogen atom [19]. 


\section{FURTHER PHYSICAL IMPLICATIONS}

We have thus far reformulated the existing mathematical devices for polarization optics in terms of the two-by-two and four-by-four representations of the Lorentz group. In so doing, we have achieved a unified group theoretical formulation of polarization optics. The next question then is whether this new formulation will lead to new applications or new experiments. This question is not unlike the question arising from Maxwell's formulation of electromagnetism. After putting together various aspects of electricity and magnetism into a single mathematical formalism, we are led to the question of whether the formalism leads to a new physics. In the case of Maxwell's equations, the new physics led to wireless communication and electronic industry.

The result of this paper is not as far-reaching as in Maxwell's case, but we are working within the same philosophical framework as the case of Maxwell's equations. Yes, we have unified various aspects of polarization optics into a single group theoretical formalism We are now interested in new conclusions which can be derived and which can be observed in laboratories. For this purpose, we note that the Lorentz group has interesting subgroups. The Lorentz group has six generators forming a closed set of commutation relations. We have already used this concept when we discussed a system consisting only of phase shifters, which is governed by $O(3)$ or the three-dimensional rotation group with three generators. The group $O(3)$ is a subgroup of the Lorentz group. It was noted that the system of attenuators is governed by the $O(2,1)$ subgroups of the Lorentz group again with three generators.

We have achieved the full six-parameter Lorentz group by combining both the phaseshifters and attenuators. We are then led to the question of whether this full Lorentz group has subgroups other than the input groups $O(3)$ and $O(2,1)$. As we discuss in Appendix A, there is an interesting subgroup which is like the two-dimensional Euclidean group. This is the product of our group theoretical formulation. In this section, we outline first the result of our effort on the Jones-matrix formalism [5]. We shall then extend this result to the case of Stokes parameters.

The Lorentz group has three boost and three rotation generators. As we shall note in Appendix A, we can construct a set of generators consisting of $J_{3}, N_{1}$ and $N_{2}$, with

$$
N_{1}=J_{1}+K_{2}, \quad N_{2}=J_{2}-K_{1} .
$$

These generators satisfy the commutation relations:

$$
\left[J_{3}, N_{1}\right]=i N_{2}, \quad\left[J_{3}, N_{2}\right]=-i N_{1}, \quad\left[N_{1}, N_{2}\right]=0 .
$$

In the case of two-by-two Jones-matrix formalism, they take the form

$$
N_{1}=\left(\begin{array}{cc}
0 & 1 \\
0 & 0
\end{array}\right), \quad N_{2}=\left(\begin{array}{cc}
0 & -i \\
0 & 0
\end{array}\right)
$$

They indeed form a closed set of commutation relations. As shown in Appendix B, these commutation relations are like those for the two-dimensional Euclidean group consisting of two translations and one rotation around the origin. This group has been studied extensively in connection with the space-time symmetries of massless particles, where $J_{1}$ and the two $N$ generators correspond to the helicity and gauge degrees of freedom respectively [20]. 
However, this group is relatively new in optics [5,21], and we are tempted to construct an optical filter possessing this symmetry. The physics of $J_{3}$ is well known through the phase shifter given in Eq.(3.4). If the angle $\delta$ is $\pi / 2$, the phase shifter becomes a quarter-wave shifter, which we write as

$$
Q=P(0, \pi / 2)=\left(\begin{array}{cc}
e^{-i \pi / 4} & 0 \\
0 & e^{i \pi / 4}
\end{array}\right) .
$$

Then $J_{1}$ and $K_{2}$ are the quarter-wave conjugates of $J_{2}$ and $K_{1}$ respectively:

$$
J_{1}=Q J_{2} Q^{-1}, \quad K_{2}=-Q K_{1} Q^{-1} \text {. }
$$

Consequently,

$$
N_{1}=Q N_{2} Q^{-1}
$$

The $N$ generators lead to the following transformation matrices.

$$
\begin{aligned}
& T_{1}(u)=\exp \left(-i u N_{1}\right)=\left(\begin{array}{cc}
1 & i u \\
0 & 1
\end{array}\right) \\
& T_{2}(v)=\exp \left(-i v N_{2}\right)=\left(\begin{array}{cc}
1 & -v \\
0 & 1
\end{array}\right) .
\end{aligned}
$$

It is clear that $T_{1}$ is the quarter-wave conjugate of $T_{2}$. We can now concentrate on the transformation matrix $T_{2}$.

If $T_{2}$ is applied to the incoming wave of Eq.(2.1),

$$
\left(\begin{array}{cc}
1 & -v \\
0 & 1
\end{array}\right)\left(\begin{array}{c}
E_{x} \\
E_{y}
\end{array}\right)=\left(\begin{array}{c}
E_{x}-v E_{y} \\
E_{y}
\end{array}\right)
$$

This new filter superposes the $y$ component of the electric field to the $x$ component with an appropriate constant, but it leaves the $y$ component invariant.

Let us examine how this is achieved. The generator $N_{2}$ consists of $J_{2}$ which generates rotations around the $z$ axis, and $K_{1}$ which generates a squeeze along the $45^{\circ}$ axis. Physically, $J_{2}$ generates optical activities. Thus, the new filter consists of a suitable combination of these two operations. In both cases, we have to take into account the overall attenuation factor. This can be measured by the attenuation of the $y$ component which is not affected by the symmetry operation of Eq. (8.8).

Is it possible to produce optical filters of this kind? Starting from an optically active material, we can introduce an asymmetry in absorption to it by either mechanical or electrical means. Another approach would be to pile up alternately the $J_{3}$-type and $K_{2}$-type layers. In either case, it is interesting to note that the combination of these two effects produces a special effect predicted from the Lorentz group.

The group $E(2)$, although new in optics, has many interesting properties having to do with our daily life. One important property is the conversion of multiplication into addition as the following matrix algebra indicates:

$$
\left(\begin{array}{cc}
1 & v_{1} \\
0 & 1
\end{array}\right)\left(\begin{array}{cc}
1 & v_{2} \\
0 & 1
\end{array}\right)=\left(\begin{array}{cc}
1 & v_{1}+v_{2} \\
0 & 1
\end{array}\right)
$$


Since this group deals with rotations and translations on a plane, it has a great potential in navigational sciences. However, we are here interested in what role this group plays in the Stokes parameters.

In the Jones-matrix formalism, we used two-by-two matrices for transformations. For the Stokes parameters, we have to use four-by-four matrices applicable to Stokes four-vectors. The four-by-four generators of the Lorentz transformations are given in Sec. IV. They are discussed in more detail in Appendix A. There, the generators $N_{1}$ and $N_{2}$ are derived from the boost and rotation generators. From these generators, we can construct transformation matrices:

$$
\begin{aligned}
T_{1}(u) & =\exp \left(-i u N_{1}\right) \\
& =\left(\begin{array}{cccc}
1+u^{2} / 2 & 0 & u & -u^{2} / 2 \\
0 & 1 & 0 & 0 \\
u & 0 & 1 & -u \\
u^{2} / 2 & 0 & u & 1-u^{2} / 2
\end{array}\right), \\
T_{2}(v) & =\exp \left(-i v N_{2}\right) \\
& =\left(\begin{array}{cccc}
1+v^{2} / 2 & -v & 0 & -v^{2} / 2 \\
-v & 1 & 0 & v \\
0 & 0 & 1 & 0 \\
v^{2} / 2 & -v & 0 & 1-v^{2} / 2
\end{array}\right) .
\end{aligned}
$$

These expressions are the four-by-four representation of the transformation matrices given in Eq.(8.7) for the Jones spinors.

Unlike the Jones-matrix formalism, the Stokes parameters can describe partially coherent light waves. This is the reason why the above four-by-four expression is complicated. These transformation matrices preserve coherence. If decoherence is introduced, we can apply the decoherence matrices discussed in Sec. VII. It is interesting to note that the Lorentz-group formulation of polarization optics opens up this kind of new possibilities in physics.

\section{CONCLUDING REMARKS}

In this paper, we have shown that both the Jones-matrix formalism and the Stokes parameters can be formulated as two different representations of the same Lorentz group. The physics of the Stokes parameters can deal with the coherence between the two polarization directions. It is shown also that the decoherence effect can also be formulated within the framework of the Lorentz group or in terms of the mathematics of squeezed states of light.

There have been in the past many laudable attempts to construct a mathematical representation for polarization optics based on the Lorentz group [17,22,23. However, the Lorentz group, particularly its relevance to optics, was not fully appreciated until it started playing the role of the underlying symmetry group for squeezed states of light [7,24, 26]. This naturally led to a new interest in possible applications of the Lorentz group in other branches of optics including polarization optics [9,10,23].

From the group theoretical point of view, what is new in this paper is that we used in Sec. $\mathrm{V}$ an additional symmetry of the Lorentz group to understand fully the connection between the Jones matrix and the Stokes parameters. This additional symmetry was the 
one which connects electrons with positrons through charge conjugation. This opens a new research line which will connect symmetries of relativistic particles with polarization optics. We can attempt to understand the symmetries of particle physics not from commutation relations of group generators but from what we observe in optics laboratories.

It is true that we used group theory as the main carrier of our analysis. On the other hand, it is true also that we did not start our paper with commutation relations, but with what we observe in the real world. We concluded this paper with what we can observe or we may possibly observe in the real world.

\section{APPENDIX A: SUBGROUPS OF THE LORENTZ GROUP}

Let us consider the space-time coordinates $(t, x, y, z)$, analogous to the Stokes parameters $\left(S_{0}, S_{1}, S_{2}, S_{3}\right)$. Then the rotation around the $z$ axis is performed by the four-by-four matrix

$$
\left(\begin{array}{cccc}
1 & 0 & 0 & 0 \\
0 & \cos \theta & -\sin \theta & 0 \\
0 & \sin \theta & \cos \theta & 0 \\
0 & 0 & 0 & 1
\end{array}\right)
$$

This transformation is generated by $J_{3}$ of Eq.(4.16). The generators of rotations around the $x$ and $y$ axes are also given in Eq.44.16) and Eq.(4.18). These three generators satisfy the closed set of commutations relations

$$
\left[J_{i}, J_{j}\right]=i \epsilon_{i j k} J_{k} .
$$

This set of commutation relations is for the three-dimensional rotation group.

The Lorentz boost along the $z$ axis takes the form

$$
\left(\begin{array}{cccc}
\cosh \eta & 0 & 0 & \sinh \eta \\
0 & 1 & 0 & 0 \\
0 & 0 & 1 & 0 \\
\sinh \eta & 0 & 0 & \cosh \eta
\end{array}\right)
$$

which is generated by $K_{3}$ of Eq.(4.20). Boosts along the $x$ and $y$ axes are generated by $K_{1}$ and $K_{2}$ given in Eq.4.17) and Eq.(4.20) respectively. These boost generators satisfy the commutation relations

$$
\left[J_{i}, K_{j}\right]=i \epsilon_{i j k} K_{k}, \quad\left[K_{i}, K_{j}\right]=-i \epsilon_{i j k} J_{k}
$$

Indeed, the three rotation generators and the three boost generators satisfy the closed set of commutation relations given in Eq.(A2) and Eq.(A4). The four-by-four transformation matrices generated by these generators are directly applicable to the space-time four-vector $(t, x, y, z)$ and also to the Stokes four-vector $\left(S_{0}, S_{1}, S_{2}, S_{3}\right)$.

We can now construct a subset of the generators consisting of $J_{3}, N_{1}$ and $N_{2}$ defined in Eq.(8.1). $J_{3}$ is given in Eq.(4.16). Thus the generators $N_{1}$ and $N_{2}$ take the form

$$
N_{1}=\left(\begin{array}{cccc}
0 & 0 & i & 0 \\
0 & 0 & 0 & 0 \\
i & 0 & 0 & -i \\
0 & 0 & i & 0
\end{array}\right), \quad N_{2}=\left(\begin{array}{cccc}
0 & -i & 0 & 0 \\
-i & 0 & 0 & i \\
0 & 0 & 0 & 0 \\
0 & -i & 0 & 0
\end{array}\right)
$$


These four-by-four matrices satisfy the closed set of commutation relations given in Eq.(8.2).

The subgroup of the Lorentz group generated by the above matrices governs the internal space-time symmetry of massless particles, and has been extensively discussed in the literature [11, 12, 15]. These expressions are new in optics.

\section{APPENDIX B: TWO-DIMENSIONAL EUCLIDEAN TRANSFORMATIONS}

In Sec. VIII, we discussed a set of commutation relations satisfied by the generators of the two-dimensional Euclidean group. The purpose of this Appendix is to construct the generators for the group of transformations on a flat plane. There are translations and rotations.

Let us consider here a two-dimensional plane and use the $x y$ coordinate system. Then $L_{z}$ defined as

$$
L_{z}=-i\left\{x \frac{\partial}{\partial y}-y \frac{\partial}{\partial x}\right\}
$$

will generate rotations around the origin. The translation generators are

$$
P_{x}=-i \frac{\partial}{\partial x}, \quad P_{y}=-i \frac{\partial}{\partial y}
$$

These generators satisfy the commutation relations:

$$
\left[L_{z}, P_{x}\right]=i P_{y}, \quad\left[L_{z}, P_{y}\right]=-i P_{x} \quad\left[P_{x}, P_{y}\right]=0 .
$$

These commutation relations are like those given in Eq.(8.2). They become identical if $L_{z}$, $P_{x}$ and $P_{y}$ are replaced by $J_{1}, N_{2}$ and $N_{3}$ respectively.

This group is not discussed often in physics, but is intimately related to our daily life. When we drive on the streets, we make translations and rotations, and thus make transformations of this $E(2)$ group. In addition, this group reproduces the internal internal space-time symmetry of massless particles [27]. This aspect of the $E(2)$ group has been extensively discussed in the literature [11, 12, 15, 20]. 


\section{REFERENCES}

[1] R. C. Jones, J. Opt. Soc. Am. 31, 488 (1941).

[2] W. A. Shurcliff, Polarized Light (Harvard Univ. Press, Cambridge, MA, 1962).

[3] E. Hecht, Am. J. Phys. 38, 1156 (1970).

[4] D. Han, Y. S. Kim, and M. E. Noz, Phys. Lett. A 206, 299 (1996).

[5] D. Han, Y. S. Kim, and M. E. Noz, J. Opt. Soc. Am. A (to be published).

[6] M. Born and E. Wolf, Principles of Optics, 6th Ed. (Pergamon, Oxford, 1980). pp 545 $-553$.

[7] Y. S. Kim and M. E. Noz, Phase Space Picture of Quantum Mechanics (World Scientific, Singapore, 1991).

[8] D. Han, Y. S. Kim, and M. E. Noz, Phys. Rev. A 37, 807 (1988).

[9] R. Y. Chiao and T. F. Jordan, Phys. Lett. A 132, 77 (1988).

[10] M. Kitano and T. Yabuzaki, Phys. Lett. A 142, 321 (1989).

[11] D. Han, Y. S. Kim, and D. Son, Am. J. Phys. 54, 818 (1986).

[12] Y. S. Kim and M. E. Noz, Theory and Applications of the Poincaré Group (Reidel, Dordrecht, 1986).

[13] I. M. Gel'fand, R. A. Minlos, and Z. Ya. Shapiro, Representations of the Rotation and Lorentz Groups and Their Applications (Pergamon, New York, 1963).

[14] D. Han, Y. S. Kim, and D. Son, J. Math. Phys. 27, 2228 (1986).

[15] S. Weinberg, Phys. Rev. 134, B882 and 135, B1049 (1964).

[16] S. Baskal and Y. S. Kim, Four-potentials and Maxwell field tensors from SL(2,C) spinors as infinite-momentum/zero-mass limits of their massive counterparts, hep-th-9512088 (1995).

[17] R. Barakat, J. Opt. Soc. Am. 53(3) 317 (1963).

[18] D. Han, Y. S. Kim, and M. E. Noz, J. Math. Phys. 36, 3940 (1995).

[19] H. C. Corben and P. Stehle, Classical Mechanics, 2nd Edition, (Wiley, New York, 1060).

[20] D. Han, Y. S. Kim, and D. Son, Phys. Rev. D 26, 3717 (1982).

[21] Y. S. Kim and L. Yeh, J. Math. Phys. 33, 1237 (1992).

[22] For early papers, see G. P. Parent and P. Roman, Nuovo Cimento 15, 370 (1960); H. Takenaka, Nouvelle Revue d'Optique 4, 37 (1973); S. R. Cloude, Optik 75, 26 (1986).

[23] For recent articles, see P. Pellat-Finet and M. Buasset, Optik (Stuttgart) 90, 101 (1992). P. Pellat-Finet, Optik (Stuttgart) 87, 27 (1991); P. Pellat-Finet and M. Buasset, ibid. 90, 101 (1992); T. Opatrny and J. Perina, Phys. Lett. A 181, 199 (1993).

[24] P. A. M. Dirac, J. Math. Phys. 4, 901 (1963).

[25] H. P. Yuen, Phys. Rev. A 13, 2226 (1976).

[26] C. M. Caves and B. L. Schumaker, Phys. Rev. A 31, 3068 (1985); B. L. Schumaker and C. M. Caves, Phys. Rev. A 31, 3093 (1985).

[27] E. Wigner, Ann. Math. 40, 149 (1939). 